NIKHEF-98-033

\title{
Reduction of multi-leg loop integrals
}

\author{
S. Weinzierl* \\ NIKHEF, P.O. Box 41882, NL - 1009 DB Amsterdam, The Netherlands
}

\begin{abstract}
I give an efficient algorithm for the reduction of multi-leg one-loop integrals of rank one. The method combines the basic ideas of the spinor algebra approach with the dual vector approach and is applicable to box integrals or higher point integrals, if at least one external leg is massless. This method does not introduce Gram determinants in the denominator. It completes an algorithm recently given by $\mathrm{R}$. Pittau.
\end{abstract}

PACS : 12.38.Bx, 11.15.Bt

Keywords : Perturbative calculations, one-loop integrals

*email address : stefanw@nikhef.nl 


\section{Introduction}

Next-to-leading order calculations have become nowadays the standard for perturbative calculations in high energy physics. One of the major obstacles is the tedious reduction of tensor loop integrals (e.g. integrals where the loop momentum appears in the numerator) to standard scalar integrals. General algorithm have been known for a long time (like the Passarino-Veltman algorithm [1], [2] or the Feynman parameter space technique [3]). Although they solve the problem in principle, the resulting expressions are usually quite long and involve the appearence of unphysical singularities in the form of Gram determinants in the denominator. These singularities usually drop out only after combination of many different structured terms.

A variety of alternative approaches exists: J.M. Campbell, E.W.N. Glover and D.J. Miller [8] introduced scalar integral functions in higher dimensions and absorbed the Gram determinants in a linear combination of these. D.B. Melrose [4] and independently W.L. van Neerven and J.A.M. Vermaseren [5] have used the fact, that in four dimensions there are maximally four linearly independent vectors, to derive a relation between a scalar pentagon integral and scalar box integrals. The derivation of van Neerven and Vermaseren was based on dual vectors. The dual vector approach was further used by G.J. van Oldenborgh and J.A.M. Vermaseren [6] and A. Signer [9]. Recently R. Pittau [7] has given an algorithm, which makes use of the spinor algebra, to reduce tensor integrals to scalar and rank one integrals. In this paper I give a formula which allows the reduction of the remaining rank one integrals to scalar integrals. It combines the basic ideas of the spinor algebra approach and the dual vector approach. This formula completes the algorithm given by R. Pittau.

This paper is organized as follows: The following section introduces the necesarry notation. In section 3 I derive the basic formula. The complete algorithm is given in section 4 . An example is worked out in section 5. Section 6 contains the conclusions.

\section{Notation}

For an introduction to spinor products I refer to [1]. A typical one-loop integral has the form

$$
I=\int \frac{d^{D} k}{(2 \pi)^{D}} \frac{\left\langle p_{1} \pm|\ldots \not k \ldots| q_{1} \pm\right\rangle \ldots\left\langle p_{m} \pm|\ldots \not k \ldots| q_{m} \pm\right\rangle}{N_{1} N_{2} \ldots N_{n}}
$$

where the $p_{i}$ and the $q_{i}$ are external massless momenta and the $N_{i}$ denote the internal propagators. The loop momentum $k$ is allowed to appear more than once in each sandwich $\left\langle p_{i} \pm|\ldots \not k \ldots| q_{i} \pm\right\rangle$. This form is general: After the decay of (possible) intermediate heavy particles all external particles can be taken to be gluons, photons or light fermions. The polarization vectors of gluons and photons are expressed in the above form using the spinor helicity method [10:

$$
\varepsilon_{\mu}^{+}(p, q)=\frac{\left\langle q-\left|\gamma_{\mu}\right| p-\right\rangle}{\sqrt{2}\langle q p\rangle}, \quad \varepsilon_{\mu}^{-}(p, q)=\frac{\left\langle q+\left|\gamma_{\mu}\right| p+\right\rangle}{\sqrt{2}[p q]}
$$

where $q$ is an arbitrary null reference momentum. It will be usefull to introduce a slight generalization of spinor products: Consider first elements of the form $\not k=a^{\mu} \gamma_{\mu}$. They form an algebra, which I will denote by $A$. It is convenient to think of the space of ket-spinors generated by the spinors obtained from all external momenta as an left $A$-module $V$. The dual space of 
bra-spinors is denoted by $V^{*}$ and is a right $A$-module. The spaces $V$ and $V^{*}$ decompose into a direct sum of subspaces of positive and negative helicity:

$$
V=V_{+} \oplus V_{-} \quad V^{*}=V_{+}^{*} \oplus V_{-}^{*}
$$

Non-vanishing spinor products are only obtained between elements of $V_{+}^{*}$ and $V_{-}$or between elements of $V_{-}^{*}$ and $V_{+}$. They are denoted by

$$
[a b]=\langle a+\mid b-\rangle \quad\langle a b\rangle=\langle a-\mid b+\rangle
$$

where $\langle a \pm| \in V_{ \pm}^{*}$ and $|b \pm\rangle \in V_{ \pm}$. Multiplication of a spinor with an element of $A$ changes the helicity, e.g. if $|p+\rangle \in V_{+}$and $\not \ell \in A$ then $\left.(\not|| p+\rangle\right) \in V_{-}$. It is convenient to define a "degree" of these objects. Assume that the spinor $|q\rangle$ corresponds to the external momentum $q$ and that

$$
|p\rangle=\phi_{n} \ldots \phi_{1}|q\rangle \text {. }
$$

Then we define $\operatorname{deg}(|q\rangle)=0$ and

$$
\operatorname{deg}(|p\rangle)=n \text {. }
$$

A similar definition applies to bra-spinors. Changing the helicity or flipping a spinor from bra to ket (or vice versa) does not change the degree. It follows that for arbitrary spinors $|p\rangle,|q\rangle$, $|k\rangle$ and $|j\rangle$

$$
\begin{aligned}
\langle q p\rangle & =-(-1)^{\operatorname{deg} p}(-1)^{\operatorname{deg} q}\langle p q\rangle, \\
{[q p] } & =-(-1)^{\operatorname{deg} p}(-1)^{\operatorname{deg} q}[p q], \\
\langle p q\rangle\langle k j\rangle & =\langle p j\rangle\langle k q\rangle+(-1)^{\operatorname{deg} q}(-1)^{\operatorname{deg} k}\langle p k\rangle\langle q j\rangle, \\
{[p q][k j] } & =[p j][k q]+(-1)^{\operatorname{deg} q}(-1)^{\operatorname{deg} k}[p k][q j] .
\end{aligned}
$$

For $\operatorname{deg} p=\operatorname{deg} q=\operatorname{deg} k=0$ these formulae coincide with the usual ones for spinor products.

I take the sign of the antisymmetric tensor as $\varepsilon_{0123}=+1$. It is useful to introduce the following short-hand notation: $\varepsilon\left(q_{a}, q_{b}, q_{c}, q_{d}\right)=4 i \varepsilon_{\mu \nu \rho \sigma} q_{a}^{\mu} q_{b}^{\nu} q_{c}^{\rho} q_{d}^{\sigma}$

\section{Derivation of the basic formula}

Combining the basic ideas of reduction methods based on dual vectors and on the spinor algebra, I derive a formula, which allows an efficient reduction of higher point integrals.

The kinematics are shown in fig.1. The $p_{i}$ are supposed to be external momenta whereas $k$ is taken as a loop momentum. I assume that the $p_{i}$ are linearly independent. This means that the method applies to pentagon or higher point integrals. A similar formula for box integrals is given later on. Let us introduce the notation

$$
\begin{aligned}
q_{i} & =\sum_{j=1}^{i} p_{i}, \\
k_{i} & =k-q_{i} .
\end{aligned}
$$

It is convenient to set $k_{0}=k$ and $p_{5}=-q_{4}$, so that one basically always deals with five external momenta $p_{1}, \ldots, p_{5}$, even for hexagon or higher point integrals. We have

$$
\begin{aligned}
& 2 k \cdot q_{i}=k^{2}-k_{i}^{2}+q_{i}^{2}, \\
& 2 k \cdot p_{i}=k_{i-1}^{2}-k_{i}^{2}+q_{i}^{2}-q_{i-1}^{2} .
\end{aligned}
$$




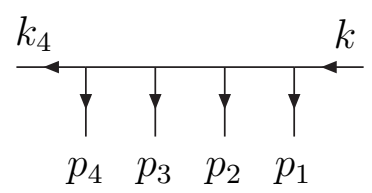

Figure 1: A subgraph of a one-loop diagram. The loop momentum is flowing through $k$ and $k_{4}$, the $p_{i}$ 's are assumed to be the external momenta.

It is convenient to work in a scheme where the Dirac matrices are in four dimensions (like the four-dimensional helicity scheme [12]). If the integral is finite we can take all vectors in four dimensions. The case of a divergent integral needs some special care: I follow the approach by G. Mahlon [13 and split the loop momentum into a four-dimensional part and a $(-2 \varepsilon)$ dimensional part

$$
k^{(D)}=k^{(4)}+k^{(-2 \varepsilon)} .
$$

It is further assumed that a four-dimensional vector is orthogonal to a $(-2 \varepsilon)$-dimensional one. I start from the dual vectors written as

$$
v_{\mu}^{a}=\frac{1}{6} \varepsilon^{a b c d} 4 i \varepsilon_{\mu \nu \rho \sigma} q_{b}^{\nu} q_{c}^{\rho} q_{d}^{\sigma},
$$

and the Schouten identity

$$
\varepsilon\left(q_{1}, q_{2}, q_{3}, q_{4}\right)\left(k^{(4)}\right)^{2}=\sum_{a=1}^{4}\left(k \cdot v^{a}\right)\left(k \cdot q_{a}\right) .
$$

This equation is related to four dimensions, therefore $k^{(4)}$ appears on the left hand side. Since $q_{a}$ and $v_{a}$ are four-dimensional objects, the scalar products of $k^{(-2 \varepsilon)}$ with $q_{a}$ or $v_{a}$ vanish and I dropped therefore the superscripts. Now

$$
\begin{aligned}
k \cdot v^{a} & = \pm \frac{1}{6} \varepsilon^{a b c d}\left(2 \operatorname{Tr}_{ \pm} \not k \phi_{b} \phi_{c} \phi_{d}-\operatorname{Tr} \not k \phi_{b} \phi_{c} \phi_{d}\right) \\
& = \pm \frac{1}{6} \varepsilon^{a b c d} 2 \operatorname{Tr}_{ \pm} \not k \phi_{b} \phi_{c} \phi_{d}
\end{aligned}
$$

where $\operatorname{Tr}_{ \pm}$denotes a trace of Dirac matrices with the insertion of a projection operator $\frac{1}{2}\left(1 \pm \gamma_{5}\right)$. Since

$$
\operatorname{Tr}\left(\not k \phi_{b} \phi_{c} \phi_{d}\right)=\left(2 k \cdot q_{b}\right)\left(2 q_{c} \cdot q_{d}\right)-\left(2 k \cdot q_{c}\right)\left(2 q_{b} \cdot q_{d}\right)+\left(2 k \cdot q_{d}\right)\left(2 q_{b} \cdot q_{c}\right)
$$

and each terms has one scalar product symmetric in two indices, these terms drop out when contracted with the antisymmetric tensor. Working out the r.h.s of eq. (12) yields

$$
\begin{aligned}
\left(k \cdot v^{a}\right)\left(k \cdot q_{a}\right)= & \pm\left(2 k \cdot q_{1} \operatorname{Tr}_{ \pm} \not k \phi_{2} \phi_{3} \phi_{4}-2 k \cdot q_{2} \operatorname{Tr}_{ \pm} \not k \phi_{1} \phi_{3} \phi_{1}\right. \\
& \left.+2 k \cdot q_{3} \operatorname{Tr}_{ \pm} \not k \phi_{1} \phi_{2} \phi_{4}-2 k \cdot q_{4} \operatorname{Tr}_{ \pm} \not k \phi_{1} \phi_{2} \phi_{3}\right)
\end{aligned}
$$


Writing $2 k \cdot q_{a}=k^{2}-k_{a}^{2}+q_{a}^{2}$, one sees that the first two terms cancel propagators, whereas the last term yields a contribution of

$$
\begin{aligned}
& \pm\left(q_{1}^{2} \operatorname{Tr}_{ \pm} \not k \phi_{2} \phi_{3} \phi_{4}-q_{2}^{2} \operatorname{Tr}_{ \pm} \not k \phi_{1} \phi_{3} \phi_{4}+q_{3}^{2} \operatorname{Tr}_{ \pm} \not k \phi_{1} \phi_{2} \phi_{4}-q_{4}^{2} \operatorname{Tr}_{ \pm} \not k \phi_{1} \phi_{2} \phi_{3}\right)= \\
& \quad= \pm\left(\operatorname{Tr}_{ \pm} \not k p_{1} p_{2} p_{3} p_{4} p_{5}+q_{1}^{2} q_{3}^{2}\left(k_{0}^{2}-k_{4}^{2}\right)+q_{1}^{2} q_{4}^{2}\left(k_{3}^{2}-k_{2}^{2}\right)+q_{2}^{2} q_{4}^{2}\left(k_{1}^{2}-k_{0}^{2}\right)\right)
\end{aligned}
$$

The r.h.s. of eq. (16) has been arranged such that no constant term survives. One finally obtains

$$
\begin{aligned}
& \operatorname{Tr}_{ \pm} \not k_{0} p_{1} p_{2} p_{3} p_{4} p_{5}=-k_{0}^{2} \operatorname{Tr}_{\mp} \not p_{1} \not p_{2} p_{3} p_{4}-k_{0}^{2} \operatorname{Tr}_{ \pm} \not k_{1} p_{2} \not p_{3} \not p_{4} \\
& +k_{1}^{2} \operatorname{Tr}_{ \pm} \not k_{0}\left(\not p_{1}+\not p_{2}\right) \not p_{3} \not p_{4}-k_{2}^{2} \operatorname{Tr}_{ \pm} \not k_{0} \not p_{1}\left(\not p_{2}+\not p_{3}\right) \not p_{4}+k_{3}^{2} \operatorname{Tr}_{ \pm} \not k_{0} \not p_{1} \not p_{2}\left(\not p_{3}+\not p_{4}\right) \\
& -k_{4}^{2} \operatorname{Tr}_{ \pm} \not k_{0} p_{1} p_{2} p_{3}-\left(k^{(-2 \varepsilon)}\right)^{2}\left(\operatorname{Tr}_{ \pm} \not p_{1} \not p_{2} p_{3} p_{4}-\operatorname{Tr}_{\mp} \not p_{1} p_{2} p_{3} p_{4}\right) \text {. }
\end{aligned}
$$

This equation holds if the Dirac matrices and the external momenta are in four dimensions. The loop momentum $k$ may be in $D$ dimensions, allowing the application also to divergent integrals. A careful inspection of the basic equation (12) shows that the term $\varepsilon\left(q_{1}, q_{2}, q_{3}, q_{4}\right)\left(k^{(4)}\right)^{2}$ on the left-hand side has to be in four dimensions, whereas the manipulations on the right-hand side involved only scalarproducts $2 k \cdot q_{a}=k^{2}-k_{a}^{2}+q_{a}^{2}$ which may be continued into $D$ dimensions as long as the external momenta $q_{a}$ stay in four dimensions. The last term of eq. (17) is a correction term, which takes care of divergent integrals. If the integral is finite, $k$ may be taken to be four dimensional and the last term vanishes. It will turn out that the correction term is irrelevant in the application of eq. (17) to pentagon or higher point integrals. The equation above may be rewritten as :

$$
\begin{aligned}
\operatorname{Tr}_{ \pm}\left(\not k p_{1} p_{2} p_{3} p_{4} p_{5}\right)= & -\operatorname{Tr}_{\mp}\left(\not p_{1} p_{2} p_{3} \not p_{4}\right)\left(N_{0}+M_{0}^{2}\right) \\
& -\frac{1}{2}\left(B_{0} \pm \varepsilon\left(k-p_{1}, p_{2}, p_{3}, p_{4}\right)\right)\left(N_{0}+M_{0}^{2}\right) \\
& +\frac{1}{2}\left(B_{1} \pm \varepsilon\left(k, p_{1}+p_{2}, p_{3}, p_{4}\right)\right)\left(N_{1}+M_{1}^{2}\right) \\
& -\frac{1}{2}\left(B_{2} \pm \varepsilon\left(k, p_{1}, p_{2}+p_{3}, p_{4}\right)\right)\left(N_{2}+M_{2}^{2}\right) \\
& +\frac{1}{2}\left(B_{3} \pm \varepsilon\left(k, p_{1}, p_{2}, p_{3}+p_{4}\right)\right)\left(N_{3}+M_{3}^{2}\right) \\
& -\frac{1}{2}\left(B_{4} \pm \varepsilon\left(k, p_{1}, p_{2}, p_{3}\right)\right)\left(N_{4}+M_{4}^{2}\right) \\
& -\left(k^{(-2 \varepsilon)}\right)^{2}\left(\operatorname{Tr}_{ \pm} p_{1} p_{2} p_{3} p_{4}-\operatorname{Tr}_{\mp} p_{1} p_{2} \not p_{3} p_{4}\right)
\end{aligned}
$$

with $N_{i}=k_{i}^{2}-M_{i}^{2}$, where the internal propagators and masses are denoted by $N_{i}$ and $M_{i}$, respectively. $B_{i}$ depends only on the pinched integral under consideration and is given by

$$
B_{i}=s t-m_{1}^{2} m_{3}^{2}-m_{2}^{2} m_{4}^{2} .
$$

Starting from the pentagon integral the internal propagator $i$ is first pinched. This gives a box integral and the value of $B_{i}$ is calculated from that box integral according to eq. (19) and according to the kinematics shown in fig. 2. Clearly the value of $B_{i}$ does not depend on the starting point for the labelling of the external legs $l_{j}$ of the box integral. Formula (19) also applies to hexagon or higher point integrals, where the sum of the external momenta from leg 


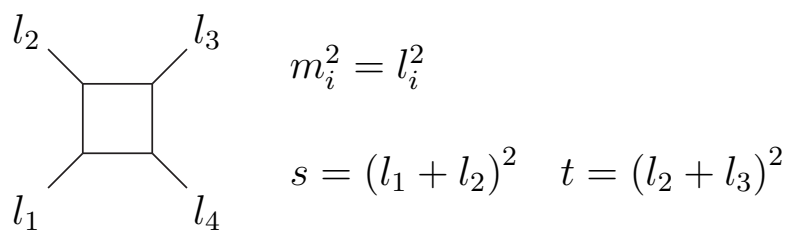

Figure 2: The labelling of a box integral with external momenta $l_{i}$.

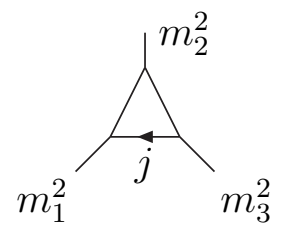

Figure 3: The labelling of a triangle integral.

5 to $\operatorname{leg} n$ is identified with $p_{5}$.

For a tensor box-integral one can use

$$
\begin{aligned}
\operatorname{Tr}_{ \pm}\left(\not k_{0} \not p_{1} p_{2} p_{3}\right)= & \frac{1}{2}\left(B \pm \varepsilon\left(k_{0}, p_{1}, p_{2}, p_{3}\right)\right) \\
& -\frac{1}{2} C_{0}\left(N_{0}+M_{0}^{2}\right)+\frac{1}{2} C_{1}\left(N_{1}+M_{1}^{2}\right) \\
& -\frac{1}{2} C_{2}\left(N_{2}+M_{2}^{2}\right)+\frac{1}{2} C_{3}\left(N_{3}+M_{3}^{2}\right)
\end{aligned}
$$

with

$$
C_{i}=m_{1}^{2}+m_{2}^{2}-m_{3}^{2}
$$

This equation is just obtained by expanding the trace. The orientation of the triangles depends on how the triangles are obtained from the box integral : If the loop momentum $k_{0}$ is not pinched, $j=k_{0}$ and $m_{1}^{2}$ is the first external mass in the direction of the flow of $j$. If $k_{0}$ is the pinched momentum, then $j=k_{1}$. The kinematics are also shown in fig.3. Equation (18) and (20) are the basic equations for the reduction of tensor loop integrals. For box integrals the terms with the antisymmetric tensor in the numerator will vanish after integration.

It remains to investigate under which condition the traces may be formed. It turns out that it is always possible to form a trace $\operatorname{Tr}_{ \pm} \not k p_{1} p_{2} p_{3} p_{4} p_{5}$ with $p_{5}=-p_{1}-p_{2}-p_{3}-p_{4}$, if at least one external leg is massless. Terms like $\langle q-|\not k| l-\rangle$, where $\mathrm{k}$ is the loop momentum, appear in the numerator of tensor integrals. Using the Schouten identity twice, one can write:

$$
[i j]\langle i j\rangle\langle q-|\not k| l-\rangle=-(-1)^{\operatorname{deg} i}(-1)^{\operatorname{deg} j}\langle q j\rangle[j l]\langle i-|\not k| i-\rangle
$$




$$
\begin{aligned}
& -(-1)^{\operatorname{deg} i}(-1)^{\operatorname{deg} j}\langle q i\rangle[i l]\langle j-|\not k| j-\rangle \\
& +\langle q j\rangle[i l]\langle i-|\not k| j-\rangle \\
& +(-1)^{\operatorname{deg} i}(-1)^{\operatorname{deg} j}\langle q i\rangle[j l]\langle i+|\not k| j+\rangle .
\end{aligned}
$$

This will be the basic equation (together with suitable choices of $i$ and $j$ ) for forming the traces. If a $n$-point integral $(n>4)$ has at least one massless external leg, say $p_{1}$, one can choose

$$
\begin{aligned}
|i-\rangle & =\left|p_{1}-\right\rangle, \\
|j-\rangle & =p_{5} p_{4} p_{3} p_{2}\left|p_{1}-\right\rangle
\end{aligned}
$$

where $p_{5}=-p_{4}-p_{3}-p_{2}-p_{1}$. Of course, if there are more massless legs, simpler choices are possible. It is easily checked by induction that the terms $\langle i-|\not k| i-\rangle$ and $\langle j-|\not k| j-\rangle$ always cancel propagators.

\section{The algorithm}

This gives us the following algorithm for the reduction of multi-leg loop integrals (e.g. at least four external legs), which is applicable if at least one external leg is massless. Three-point and two-point integrals are usually not so complicated and can be reduced using standard techniques.

Step 1 consists in reducing tensor integrals to rank one integrals and was given by R. Pittau [7]: If there at least two massless legs, say $p_{1}$ and $p_{2}$, one writes

$$
\not k=\frac{1}{2 p_{1} \cdot p_{2}}\left[\left(2 k \cdot p_{2}\right) \not p_{1}+\left(2 k \cdot p_{1}\right) \not p_{2}-\not p_{1} \not k p_{2}-\not p_{2} \not k p_{1}\right] \text {. }
$$

The first two terms already cancel propagators, whereas a product of the last two terms can be rewritten as

$$
\begin{aligned}
\langle 1-|\not k| 2-\rangle\langle 2-|\not k| 1-\rangle= & \left(2 k \cdot p_{1}\right)\left(2 k \cdot p_{2}\right)-\left(2 p_{1} \cdot p_{2}\right) k^{2}, \\
\langle 1-|\not k| 2-\rangle\langle 2+|\not k| 1+\rangle= & \frac{1}{\langle 2-|3| 1-\rangle}\left[\left(2 p_{1} \cdot p_{3}\right)\left(2 p_{2} \cdot k\right)\langle 1-|\not k| 2-\rangle\right. \\
& -\left(2 p_{1} \cdot k\right)\left\langle 1-\left|\not p_{2} \not k p_{3}\right| 2-\right\rangle \\
& +\left(2 p_{3} \cdot k\right)\left\langle 1-\left|\not p_{2} \not k p_{1}\right| 2-\right\rangle \\
& \left.+k^{2}\left\langle 1-\left|p_{2} p_{3} \not p_{1}\right| 2-\right\rangle\right],
\end{aligned}
$$

where $p_{3}$ is another (not necessarily massless) external leg. If there are no massless legs, or only one massless leg, one can take linear combinations of the external momenta in order to form two massless momenta [0]. If all external momenta are massive this will re-introduce Gram determinants.

In step 2 we reduce the remaining rank one integrals to scalar integrals by first forming the trace with the help of equation (22). This can always be done if at least one external leg is massless. Using equation (18) and equation $(20)$ we then obtain scalar integrals, integrals with an antisymmetric tensor in the numerator and a correction term. For box integrals the terms 


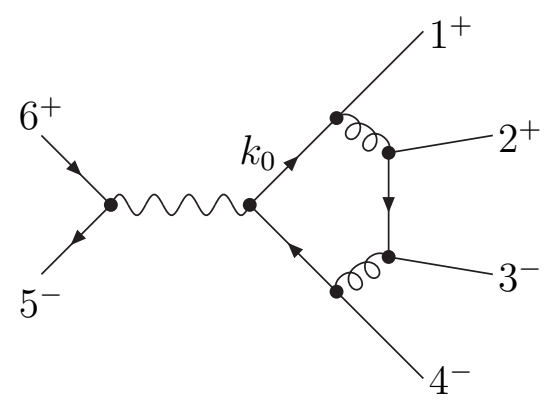

Figure 4: The Feynman diagram with the helicity assignment. All external momenta are taken to be outgoing.

with the antisymmetric tensor in the numerator will vanish after integration. For higher point integrals one may rewrite

$$
\varepsilon\left(k, p_{1}, p_{2}, p_{3}\right)=\operatorname{Tr}_{+}\left(\not k p_{1} \not p_{2} p_{3}\right)-\operatorname{Tr}_{-}\left(\not k \not p_{1} \not p_{2} \not p_{3}\right)
$$

and iterate the procedure.

Step 3 : The correction term is evaluated as follows [14]:

$$
\int \frac{d^{4-2 \varepsilon} k}{(2 \pi)^{4-2 \varepsilon}} \frac{\left(k^{(-2 \varepsilon)}\right)^{2}}{N_{1} N_{2} \ldots N_{n}}=\varepsilon(4 \pi) \int \frac{d^{6-2 \varepsilon} k}{(2 \pi)^{6-2 \varepsilon}} \frac{1}{N_{1} N_{2} \ldots N_{n}}
$$

The correction term is proportional to the scalar integral in $D=6-2 \varepsilon$ dimensions. These integrals are finite in the limit $\varepsilon \rightarrow 0$ for $n \geq 4$ [3] and the correction term does therefore not contribute to order $O\left(\varepsilon^{0}\right)$.

\section{An example}

As an example I consider here a pentagon integral which was recently encountered in the oneloop calculation for $e^{+} e^{-} \rightarrow q \bar{q} Q \bar{Q}$ [15]. We want to evaluate the helicity diagram shown in fig. ๒. The diagram corresponds to the following integral:

$$
I=-8 \frac{[12]\langle 34\rangle}{s_{56}} \int \frac{d^{D} k}{(2 \pi)^{D}} \frac{\left\langle 5-\left|\not k_{4} \not k_{2} \not k_{0}\right| 6-\right\rangle}{k_{0}^{2} k_{1}^{2} k_{2}^{2} k_{3}^{2} k_{4}^{2}}
$$

The numerator is rewritten as

$$
\begin{aligned}
\left\langle 5-\left|\not \not_{4} \not \not_{2} \not \not_{0}\right| 6-\right\rangle= & -\left\langle 5-\left|\left(\not p_{3}+\not p_{4}\right) \not k_{2}\left(\not p_{1}+\not p_{2}\right)\right| 6-\right\rangle \\
& +k_{2}^{2}\left\langle 5-\left|\not k_{0}\right| 6-\right\rangle-k_{2}^{2}\left\langle 5-\left|\not p_{3}+\not p_{4}\right| 6-\right\rangle
\end{aligned}
$$

The first term on the r.h.s of eq. (29) corresponds to a rank one pentagon integral, the second term to a rank one box integral, whereas the last term already corresponds to a scalar box 
integral. The rank one pentagon integral is reduced as follows: Multiplying with [23] $\langle 23\rangle$ and using eq. (22) we obtain:

$$
\begin{aligned}
{[23]\langle 23\rangle\left\langle 5-\left|\left(\not p_{3}+\not p_{4}\right) \not k_{2}\left(\not p_{1}+\not p_{2}\right)\right| 6-\right\rangle=} \\
=\quad-\langle 6+|1+2| 3+\rangle[34]\langle 45\rangle\left(k_{1}^{2}-k_{2}^{2}\right)-[61]\langle 12\rangle\langle 2+|3+4| 5+\rangle\left(k_{2}^{2}-k_{3}^{2}\right) \\
\quad+\frac{\langle 6+|1+2| 3+\rangle\langle 2+|3+4| 5+\rangle}{\langle 34\rangle\langle 4+|5+6| 1+\rangle[12]} \operatorname{Tr}_{+}\left(\not k_{2} \not p_{3} \not_{4}\left(\not p_{5}+\not p_{6}\right) \not p_{1} p_{2}\right) \\
\quad+\frac{[61]\langle 45\rangle}{\langle 4-|5+6| 1-\rangle} \operatorname{Tr}_{-}\left(\not k_{2} p_{3} \not p_{4}\left(\not p_{5}+\not p_{6}\right) \not p_{1} \not p_{2}\right) .
\end{aligned}
$$

According to eq. (18) the traces give after integration

$$
\begin{aligned}
& \int \frac{d^{D} k}{(2 \pi)^{D}} \frac{\operatorname{Tr}_{ \pm}\left(\not k_{2} p_{3} p_{4}\left(\not p_{5}+\not p_{6}\right) \not p_{1} p_{2}\right)}{k_{0}^{2} k_{1}^{2} k_{2}^{2} k_{3}^{2} k_{4}^{2}}=\int \frac{d^{D} k}{(2 \pi)^{D}} \frac{1}{k_{0}^{2} k_{1}^{2} k_{2}^{2} k_{3}^{2} k_{4}^{2}}\left(-k_{2}^{2} \operatorname{Tr}_{\mp}\left(\not p_{3} p_{4}\left(\not p_{5}+\not p_{6}\right) \not p_{1}\right)\right. \\
& \left.\quad-\frac{1}{2}\left(s_{123} s_{234}-s_{23} s_{56}\right) k_{2}^{2}+\frac{1}{2} s_{12} s_{234} k_{3}^{2}-\frac{1}{2} s_{12} s_{23} k_{4}^{2}+\frac{1}{2} s_{23} s_{34} k_{0}^{2}-\frac{1}{2} s_{123} s_{34} k_{1}^{2}\right) \\
& \quad+O(\varepsilon)
\end{aligned}
$$

The rank one box integral is evaluated as follow: After multiplication with [14] $\langle 14\rangle$ we obtain

$$
\begin{aligned}
{[14]\langle 14\rangle\left\langle 5-\left|\not k_{0}\right| 6-\right\rangle=} \\
=-\langle 54\rangle[46]\left(k_{0}^{2}-k_{1}^{2}\right)-\langle 51\rangle[16]\left(k_{3}^{2}-k_{4}^{2}+s_{56}-s_{123}\right) \\
\quad+\frac{\langle 54\rangle[16]}{\langle 4-|2+3| 1-\rangle} \operatorname{Tr}_{+}\left(\not k_{0} p_{1}\left(\not p_{2}+\not p_{3}\right) \not p_{4}\right) \\
\quad+\frac{\langle 51\rangle[46]}{\langle 4+|2+3| 1+\rangle} \operatorname{Tr}_{-}\left(\not k_{0} p_{1}\left(\not p_{2}+\not p_{3}\right) \not p_{4}\right)
\end{aligned}
$$

The traces give after integration (using eq. (20)):

$$
\begin{aligned}
\int \frac{d^{D} k}{(2 \pi)^{D}} \frac{\operatorname{Tr}_{ \pm}\left(\not k_{0} \not p_{1}\left(\not p_{2}+\not p_{3}\right) \not p_{4}\right)}{k_{0}^{2} k_{1}^{2} k_{3}^{2} k_{4}^{2}}= & \frac{1}{2} \int \frac{d^{D} k}{(2 \pi)^{D}} \frac{1}{k_{0}^{2} k_{1}^{2} k_{3}^{2} k_{4}^{2}}\left(s_{123} s_{234}-s_{23} s_{56}\right. \\
& -\left(s_{23}-s_{234}\right) k_{0}^{2}+\left(s_{56}-s_{123}\right) k_{1}^{2} \\
& \left.-\left(s_{56}-s_{234}\right) k_{3}^{2}+\left(s_{23}-s_{123}\right) k_{4}^{2}\right)
\end{aligned}
$$

This completes the reduction to scalar integrals. In this example all propagators were massless. The extension to massive lines is straightforward. If for example $k_{2}$ is replaced by a massive line, the algorithm yields a combination of scalar boxes and a scalar pentagon integral. The scalar pentagon integral can be expressed in terms of scalar boxes according to well-known formulae [3, 5].

\section{Conclusions}

In this paper, I have given a formula which allows the reduction of rank one pentagon or higher point integrals. If there is at least one massless external leg, this formula can always be applied. This method does not introduce a Gram determinant in the denominator. A corresponding formula for rank one box-integrals was also given. For rank one triangles or bubbles conventional 
techniques are usually efficient enough. The formulae in this paper complete an algorithm recently given by R. Pittau, and form together an efficient algorithm for reducing tensor loop integrals.

I would like to thank D.A. Kosower and J.A.M. Vermaseren for useful discussions and for suggestions concerning the manuscript.

\section{References}

[1] G.Passarino and M.Veltman, Nucl.Phys. B160, (1979), 151

[2] R.G. Stuart, Comp. Phys. Comm. 48, (1988), 367

R.G. Stuart and A. Góngora-T., Comm. Phys. Comm. 56, (1990), 337

[3] Z.Bern, L.Dixon and D.A.Kosower, Nucl.Phys. B412, (1994), 751

[4] D.B.Melrose, Nuovo Cimento 40A, (1965), 181

[5] W.L.van Neerven and J.A.M.Vermaseren, Phys.Lett. 137B, (1984),241

[6] G.J. van Oldenborgh and J.A.M.Vermaseren, Z.Phys. C46, (1990), 425

[7] R.Pittau, Comput.Phys.Commun. 104, (1997), 23

R.Pittau, Comput.Phys.Commun. 111, (1998), 48

[8] J.M.Campbell, E.W.N.Glover and D.J.Miller, Nucl. Phys. B498, (1997), 397

[9] A Signer, Ph.D. thesis, Diss. ETH Nr. 11143

[10] P. De Causmaecker, R.Gastmans, W.Troost and T.T.Wu, Nucl.Phys. B206, (1982), 53

R.Kleiss and W.J.Stirling, Nucl.Phys. B262, (1985), 235

J.F.Gunion and Z.Kunzst, Phys.Lett. 161B, (1985), 333

Z.Xu, D.-H.Zhang and L.Chang, Nucl.Phys. B291, (1987), 392

[11] M.Mangano and S.Parke, Phys.Rep. 200, (1991), 301

L.Dixon, TASI 1995, hep-ph/9601359

[12] Z.Bern and D.A.Kosower, Nucl.Phys. B379, (1992), 451

[13] G.Mahlon, Phys.Rev. D49, (1994), 2197

G.Mahlon, Phys.Rev. D49, (1994), 4438

[14] Z.Bern and A.G.Morgan, Nucl.Phys. B467, (1996), 479

[15] Z.Bern, L.Dixon, D.A.Kosower and S.Weinzierl, Nucl.Phys. B489, (1997), 3 